\title{
Nonanalytic enhancement of the charge transfer from adatom to one-dimensional semiconductor superlattice and optical absorption spectrum
}

\author{
Satoshi Tanaka,,$*$ Sterling Garmon, ${ }^{2}$ and Tomio Petrosky $2,3,+$ \\ ${ }^{1}$ Department of Physical Science, Osaka Prefecture University, Sakai 599-8531, Japan \\ ${ }^{2}$ Center for Studies in Statistical Mechanics and Complex Systems, \\ The University of Texas at Austin, Austin, TX 78712 USA \\ ${ }^{3}$ International Solvey Institute for Physics and Chemistry, CP231, 1050 Brussels, Belgium
}

(Dated: September 17, 2018)

\begin{abstract}
The charge transfer from an adatom to a semiconductor substrate of one-dimensional quantum dot array is evaluated theoretically. Due to the Van Hove singularity in the density of electron states at the band edges, the charge transfer decay rate is enhanced nonanalytically in terms of the coupling constant $g$ as $g^{4 / 3}$. The optical absorption spectrum for the ionization of a core level electron of the adatom to the conduction band is also calculated. The reversible non-Markovian process and irreversible Markovian process in the time evolution of the adatom localized state manifest themselves in the absorption spectrum through the branch point and pole contributions, respectively.
\end{abstract}

*Electronic address: stanaka@p.s.cias.osakafu-u.ac.jp

${ }^{\dagger}$ Electronic address: petrosky@physics.utexas.edu 


\section{INTRODUCTION}

Thanks to recent advances in nanotechnology, various types of artificial low-dimensional semiconductor structures have been fabricated [1]. The quantum confinement of electron in these structures greatly modifies the density of states of carriers resulting in complete different electronic and optical properties from the bulk system [2, 3]. Recently one dimensional quantum wire and quantum-dot array have been manufactured in various ways [1, 4], and the formation of one dimensional miniband has been theoretically and experimentally investigated [5, 6, 7]. It has been found that the Van Hove singularity of the density of state inherent with the one-dimensionality causes a characteristic electronic transport [8, 9].

In this paper, we consider the charge transfer between an adatom localized state and a one dimensional miniband associated with a quantum wire or quantum-dot array. The charge transfer between an adatom and a substrate semiconductor has been extensively studied [10]. We will show that the low dimensionality greatly modifies the charge transfer process from the adatom to the semiconductor quantum dot array due to the singularity of the density of states. The physical situation we consider in this paper is shown in Fig. 11(a) where an adatom is attached to a semiconductor quantum-dot array surface (hereafter we simply call it a superlattice).

The charge transfer of an electron from adatom to the miniband of the superlattice is caused by the hybridization of the adatom wave function with the miniband which can be described by the bilinear coupling between the adatom localized state and the bound state of a single quantum dot in which the adatom is situated. The situation may be described by one-dimensional version of Newns-Anderson model which has been extensively used to investigate the charge transfer process between the adatom and the semiconductor substrate [10,11]. As shown below, the one-dimensional Newns Anderson model we consider here is equivalent to the Friedrichs Hamiltonian which we have investigated in our previous letter [12].

In this recent letter we reported a vast increase in the decay rate of an excited dipole molecule traveling in an one-dimensionally confined electromagnetic waveguide when the

cutoff frequency of the waveguide is near the characteristic frequency of the dipole [12]. This vast increase is a direct consequence of a singularity in the density of photon states at the cutoff frequency. Due to this singularity, standard perturbation analysis breaks down 
and hence one cannot apply Fermi's golden rule to evaluate the decay rate in the vicinity of the singularity. We have shown that in this case the decay rate of the excited dipole depends non-analytically as $g^{4 / 3}$ on the coupling constant $g$. In the present article, we report that the same nonanalytic enhancement of the decay rate (including the $g^{4 / 3}$ law) can be found in our system, despite the fact that the density of electron states of the miniband of the superlattice is different from the density of states of photon states in a waveguide.

Although the exponential decay law for the unstable state has been observed ubiquitously in nature, quantum mechanics predicts that there should be a deviation from the exponential decay law for the unstable state [13]. It has been shown that, irrespective of any specific form for the interaction, the time evolution of the surviving amplitude of an unstable state deviates from the exponential law on short and long time scales due to the existence of the lower bound on the energy, i.e., the branch point effect. Wilkinson et al. has recently succeeded in measuring the branch point effect in super-cooled sodium atoms [14], even though the timescale in which the deviation from the exponential decay law appears is very short, Thus separation of the pole and branch point effects in the time evolution of the unstable state is essential to our understanding of the decay process, as has been done for a system composed of an excited atom coupled to a radiation field [15, 16]. The separation of the two effects is also useful because the reversible non-Markovian process in the decay process (due to the branch point effect) is directly related to the quantum Zeno effect [17, 18, 19, 20].

Our goal is to present an actual experimental method that enables us to separately evaluate the pole and branch point effects for the decay process of the unstable state in this system. In this article, we consider the optical absorption process in which a core electron of the adatom with discrete energy is transferred to the continuous conduction band. It will be shown here that the spectral shape is influenced by the Markovian process due to the pole effect and also by the non-Markovian process due to the branch point effect.

The Friedrichs model presented here is equivalent to that known as the Fano model which was originally developed to explain the absorption spectrum for the autoionization process in the He atom [22]. It is well known that this absorption spectrum has an asymmetric spectral profiles due to quantum interference between different optical transition paths. The appearance of the quantum interference indicates that the quantum coherence, which is a source of the memory effect, plays a key role in the decaying process of the excited state. As will be shown in the present paper, since the branch point effect which accounts for the 
non-Markovian decaying process with a memory effect is intensified by the singularity in the density of states, the absorption spectral profile provides us with the information on the extent of the persistence of the quantum coherence in the decaying process.

In $\S 2$ we present the model and show the nonanalytic enhancement of the decay rate for the unstable state. We decompose the absorption spectrum into contributions from the pole and branch point effects in $\S 3$. In $\S 4$ we summarize our results and provide some discussion.

\section{MODEL AND NONANALYTIC ENHANCEMENT OF DECAY RATE}

We consider a 1D semiconductor superlattice with an adatom on the surface, as shown in Fig प(a). The width of each potential well is a few $\mathrm{nm}$ to $100 \mathrm{~nm}$. One can make this

device, for example, with GaAs/GaAlAs heterostructures [1, 5]. The superlattice consists of $N \gg 1$ identical quantum wells, and each well is assumed to have a single bound state $|\tilde{n}\rangle$ $(\tilde{n}=-N$ to $N)$ of equal energy, where the tilde is used to distinguish the site representation from the wavenumber representation below. A miniband is formed in the superlattice due to the electron tunneling through the potential barrier [2, 6, 7, 23]. We assume only nearest neighbor tunneling occurs with a transition probability of $-B / 2$. The $1 \mathrm{D}$ superlattice is then represented by the one-dimensional tight binding model, and we have a continuous miniband of width $2 B$ in the limit of $N \rightarrow \infty$. In addition to the miniband we consider an adatom localized state $|d\rangle$ with energy $E_{0}$; also we consider an inner core level $|c\rangle$ with energy $E_{c}$. Both of these states are associated with the adatom impurity located at the $n=0$ site. The adatom localized state $|d\rangle$ is hybridized with the $|\tilde{0}\rangle$ state with coupling strength $g B$.

Taking $\hbar=1$ hereafter, the electronic Hamiltonian $H_{E}$ is then written as

$$
\begin{aligned}
H_{E}= & E_{c}|c\rangle\left\langle c\left|+E_{0}\right| d\right\rangle\left\langle d\left|-\frac{B}{2} \sum_{<m, n>}\right| \tilde{m}\right\rangle\langle\tilde{n}| \\
& +g B(|d\rangle\langle\tilde{0}|+| \tilde{0}\rangle\langle d|),
\end{aligned}
$$

where $\langle\cdots\rangle$ means taking nearest neighbor sum in Eq.(11). By introducing the wavenumber representation

$$
|k\rangle \equiv \frac{1}{\sqrt{N}} \sum_{n=1}^{N} \exp [i k n]|\tilde{n}\rangle
$$


we can rewrite $H_{E}$ in the form of the Friedrichs model

$$
\begin{gathered}
H_{E}=E_{c}|c\rangle\left\langle c\left|+E_{d}\right| d\right\rangle\left\langle d\left|+\sum_{k} E_{k}\right| k\right\rangle\langle k| \\
+\frac{1}{\sqrt{N}} \sum_{k} g B(|d\rangle\langle k|+| k\rangle\langle d|),
\end{gathered}
$$

where $E_{k}=-B \cos k$. We impose periodic boundary conditions, leading to $k=2 \pi j / N$, where $j$ is an integer running from $-N / 2$ to $N / 2$. The energy dispersion relation gives a divergence in the density of states at either band edge:

$$
\rho\left(E_{k}\right)=\frac{1}{\pi} \frac{1}{\sqrt{B^{2}-E_{k}^{2}}} .
$$

For the 1D Friedrichs model, the electronic Hamiltonian $H_{E}$ is diagonalized in terms of the so-called Friedrichs solution which results in the spectral representation 21]

$$
H_{E}=E_{c}|c\rangle\left\langle c\left|+\sum_{i=1}^{2} E_{i}\right| \phi_{i}\right\rangle\left\langle\phi_{i}\left|+\sum_{k} E_{k}\right| \phi_{k}^{F}\right\rangle\left\langle\phi_{k}^{F}\right|,
$$

where $\left|\phi_{i}\right\rangle(i=1,2)$ are two stable eigenstates with energies $E_{i}$, and $\left|\phi_{k}^{F}\right\rangle$ with energy $E_{k}$ (where $\left|E_{k}\right|<B$ ) is given by

$$
\begin{aligned}
\left|\phi_{k}^{F}\right\rangle & =|k\rangle+\frac{1}{\sqrt{N}} \frac{g B}{\eta^{+}\left(E_{k}\right)} \\
& \times\left(|d\rangle+\frac{1}{\sqrt{N}} \sum_{k^{\prime}(\neq k)} \frac{g B}{E_{k}-E_{k^{\prime}}+i \epsilon}\left|k^{\prime}\right\rangle\right),
\end{aligned}
$$

with

$$
\begin{aligned}
& \frac{1}{\eta^{+}(z)}=G_{d d}^{+}(z) \equiv\left\langle d\left|\frac{1}{\left(z-H_{E}\right)^{+}}\right| d\right\rangle \\
& =\frac{1}{\left[z-E_{0}-\frac{1}{N} \sum_{k} \frac{g^{2} B^{2}}{z-E_{k}}\right]^{+}}
\end{aligned}
$$

and a positive infinitesimal $\epsilon$. The Green function $G_{d d}^{+}(z)$ is analytically continued from the upper half complex $E_{k}$ plane to the lower half plane. Here, we do not write explicit forms of $\left|\phi_{i}\right\rangle$, since we will not use them in this paper [21]. In the limit of $N \rightarrow \infty$, the summation over the wavenumber turns to the continuous integral, and then the self-energy term in Eq.(7) reads

$$
\begin{aligned}
& \Xi(z) \equiv \frac{1}{N} \sum_{k} \frac{g^{2} B^{2}}{z-E_{k}} \rightarrow \frac{1}{2 \pi} \int_{-\pi}^{\pi} d k \frac{g^{2} B^{2}}{z-E_{k}} \\
& =\int_{-B}^{B} d E_{k} \rho\left(E_{k}\right) \frac{g^{2} B^{2}}{z-E_{k}}=\frac{g^{2} B^{2}}{\sqrt{z^{2}-B^{2}}} .
\end{aligned}
$$


By substituting the right-hand side of Eq.(8) into $\eta^{+}(z)$ and taking the square of the dispersion relation we obtain a quartic equation in $z$. From the form of the quartic equation, it is readily seen that the solutions are symmetric about the origin $E_{0}=0$. By applying the standard method for solving a quartic equation, one can find the explicit solutions of the dispersion equation $\eta^{+}(z)=0$. The solutions consist of the pole $z_{d} \equiv \tilde{E}_{0}-i \gamma$ of $G_{d d}^{+}(z)$ in the lower half complex plane in the second Riemann sheet corresponding to the unstable decaying state, and the poles on the real axis corresponding to the stable states, $E_{i}(i=1,2)$. For illustration, we plot in Fig.2(a) $\tilde{E}_{0}$ and $E_{i}$ for $g=0.5$ as a function of $E_{0} / B$ with thick dashed and solid lines, respectively. For arbitrary $E_{0}$, there always exist two stable solutions outside the electronic miniband. Note that there is a critical value $E_{\gamma}$ at which the unstable state with imaginary part $\gamma \neq 0$ appears. The unstable state exists for all $\left|E_{0}\right|<E_{\gamma}$, as indicated by the arrows in Fig[2(a). In Fig. 2(b) we plot the decay rate $\gamma / B$ of the unstable solution for $g=0.1$ as a function of $E_{0} / B$. The maximum value of $\gamma=\gamma_{\max }$ occurs at $E_{0}= \pm B$.

Since the explicit form of the full solution is complicated, we present an approximate calculation of the maximum value $\gamma_{\max }$ of $\gamma$ at $E_{0} / B= \pm 1$ and the critical value $E_{\gamma}$ of $E_{0}$ where the unstable solution appears. To estimate $\gamma_{\max }$ we put $E_{0}=B$ in the dispersion equation. After a simple manipulation we obtain

$$
\zeta=1+(-1)^{2 / 3} \frac{g^{4 / 3}}{(\zeta+1)^{1 / 3}},
$$

where $\zeta \equiv z / B$. The zeroth order solution (for $g=0$ ) is $\zeta=1$. We use this as our starting point and solve iteratively to find

$$
\frac{\gamma_{\max }}{B}=\frac{\sqrt{3} g^{4 / 3}}{2^{4 / 3}}+\frac{g^{8 / 3}}{2^{8 / 3} \sqrt{3}}+O\left(g^{16 / 3}\right),
$$

where the third order contribution $\left(g^{4 j / 3}\right.$ with $\left.j=3\right)$ vanishes.

To estimate $E_{\gamma}$ near $E_{0}=B$ we put $z=E_{0}+g^{\alpha} z_{1}$ where $\alpha>0$ and $z_{1}$ are unknown variables which are independent of $g$. Then, by keeping only the predominant contribution to the dispersion equation, we obtain

$$
\zeta-\bar{E}_{0} \approx \frac{g^{2}}{\sqrt{\bar{E}_{0}+1} \sqrt{\zeta-1}}
$$

where $\bar{E}_{0} \equiv E_{0} / B$. Squaring this equation yields

$$
f(\zeta)=\left(\zeta-\bar{E}_{0}\right)^{2}(\zeta-1)-\frac{g^{4}}{\bar{E}_{0}+1} \approx 0 .
$$


The function $f(\zeta)$ represents a cubic curve. By taking the derivative of $f(\zeta)$ and setting $f^{\prime}(\zeta)=0$, we find the threshold value of $\bar{E}_{0}=E_{\gamma} / B>1$ at which the complex solutions of $f(\zeta)=0$ appear. This leads to the first two terms in the following expression for $E_{\gamma}$ :

$$
\frac{E_{\gamma}}{B}=1+\frac{3 g^{4 / 3}}{2}-\frac{g^{8 / 3}}{8}+O\left(g^{12 / 3}\right)
$$

For precision, we have presented the second order correction that is obtained from the exact solution of the original quartic equation. The values of $\gamma$ and $E_{\gamma}$ are non-analytic in $g$ for $g=0$, and hence one cannot obtain these results from a series expansion in $g$ using ordinary perturbation analysis.

It should be noted that the $g^{4 / 3}$ law for the decay rate is rather universal around the edge of the continuous spectrum. Indeed, if the integration over the wavenumber in the selfenergy is a Cauchy integral that is a double-valued function on $z$, as is the case in Eq.(7), then the edge of the miniband will give a square root type of essential singularity in the self-energy. In addition, if this singularity leads to a divergence at the edge, one can show that the $g^{4 / 3}$ effect appears in the vicinity of the singularity. Indeed, this is also the case for the dipole molecule traveling inside the waveguide mentioned above [12].

In terms of the solutions of the dispersion equation obtained here, we may evaluate the time evolution of the surviving amplitude of the adatom localized state defined by $P(t) \equiv$ $\left|\left\langle d\left|\exp \left[-i H_{E} t\right]\right| d\right\rangle\right|^{2}$. Indeed, aside from the persistent oscillation attributed to the two stable states, there are two contributions to the time evolution of the surviving amplitude; one comes from the pole contribution over the wavenumber integral, where the location of the pole is given by the solution of the dispersion relation $\eta^{+}\left(E_{k}\right)=0$ in the complex $E_{k}$ plane discussed above, and the other comes from the two branch point contributions that are located at the edges of the miniband at $\pm B$. As usual, the pole contribution leads to an exponential decay $\exp \left[-i\left(\tilde{E}_{0}-i \gamma\right) t\right]$, while the branch point leads to a power law decay. If the condition $1-\left|E_{0}\right| / B \gg g$ is satisfied, we have a well separated time scale between the exponential and power law decay in the decay process of the localized state. The exponential law for the decay process is thus a good approximation for a time scale on the order of $t \sim 1 / \gamma$. However, once this condition is no longer fulfilled, the time separation becomes obscure and we must take into account the branch point effects in the time evolution. It is, however, a cumbersome task to evaluate the integral associated with the branch point in the time evolution. In the next section, we will evaluate the optical 
absorption spectrum instead, to separate the contribution of the Markovian effect from the non-Markovian effect. It should be generally easier in experiment to observe the absorption spectrum than a time evolution of the state $|d\rangle$.

\section{ABSORPTION SPECTRUM}

\section{A. Spectral representation and the separation of the pole and branch point con-} tributions

Here we consider the optical absorption by a transition from an inner core level of the adatom to the conduction electronic states of the 1D semiconductor superlattice, as shown in Fig!(b). There are two optical transition paths from the core level $|c\rangle$ with large transition amplitudes: One is that from the core level $|c\rangle$ to the localized state $|d\rangle$ and the other is from the core level $|c\rangle$ to the $\tilde{n}=0$ site $|\tilde{0}\rangle$. The transition probabilities are denoted by $T_{d c}$ and $T_{0 c}$, respectively. The contributions of the transitions to the other bound states $|\tilde{n}\rangle$ are small, so we neglect the contributions in this paper, though it is not difficult to include those effects.

We consider here a single mode of the optical light field with frequency $\Omega$. The total Hamiltonian is then written as

$$
H_{\text {total }}=H_{R}+H_{E}+H_{R E}
$$

where the electronic Hamiltonian $H_{E}$ is given in Eq.(3) and

$$
\begin{aligned}
H_{R} & =\Omega a^{\dagger} a \\
H_{R E} & =\left(T_{d c}|d\rangle\left\langle c\left|+T_{0 c}\right| \tilde{0}\right\rangle\langle c|\right) a+\text { H.c. } \\
& \equiv \hat{T} a+a^{\dagger} \hat{T}^{\dagger} .
\end{aligned}
$$

The $H_{R}$ represents a monochromatic light field where $a\left(a^{\dagger}\right)$ is the annihilation (creation) operator of the field, and $H_{R E}$ describes the interaction between the light field and the electronic system under the rotating wave approximation.

In the initial state of the absorption process, we have a core electron in the $|c\rangle$ state and a single photon with energy $\Omega$; we write this initial state as $|c ; 1\rangle$ with energy $E_{c}+\Omega$, where 1 denotes the photon number for the mode $\Omega$. After the absorption process the energy of 
the photon is transferred to the electronic system. The final states then take the form $\left|\phi_{i} ; 0\right\rangle$ $(i=1,2)$ or $\left|\phi_{k}^{F} ; 0\right\rangle$, with the energies $E_{i}$ or $E_{k}$ respectively.

When the value of $E_{c}+\Omega$ falls in between $-B$ and $B$, the state $|c ; 1\rangle$ is resonant with the electronic continuum $\left|\phi_{k}^{F}\right\rangle$. As a result, the energy transfer from the light field to the electronic system occurs. Hereafter we take $-E_{c}$ as the origin of the light energy: $\Omega+E_{c} \rightarrow \Omega$.

The decay rate of $|c ; 1\rangle$ is then determined by the pole location of the Green's function for $|c ; 1\rangle$ as

$$
G_{c c}^{+}(z) \equiv\left\langle c ; 1\left|\frac{1}{\left[z-H_{\text {total }}\right]^{+}}\right| c ; 1\right\rangle .
$$

The decay rate $\gamma_{a b s}(\Omega)$ is a function of $\Omega$, and thus we identify the absorption spectrum as $F(\Omega) \equiv \gamma_{a b s}(\Omega)$. In the weak coupling limit of $T_{d c}$ and $T_{0 c}$, the absorption spectrum $F(\Omega)$ reduces to

$$
\begin{aligned}
& F(\Omega)=-\lim _{\epsilon \rightarrow 0+} \operatorname{Im} \sum_{k} \frac{\left|\left\langle\phi_{k}^{F}|\hat{T}| c\right\rangle\right|^{2}}{\Omega-E_{k}+i \epsilon} \\
& =-\lim _{\epsilon \rightarrow 0+} \operatorname{Im} \sum_{k} \frac{\mid\left\langle\phi_{k}^{F}\left|\left(T_{d c}|d\rangle+T_{0 c}|\tilde{0}\rangle\right)\right|^{2}\right.}{\Omega-E_{k}+i \epsilon} .
\end{aligned}
$$

The matrix element in Eqs.(17) may be obtained by using Eq.(6) :

$$
\begin{aligned}
\left\langle d \mid \phi_{k}^{F}\right\rangle & =\frac{g B}{\sqrt{N}} \frac{1}{\eta^{+}(z)} \\
\left\langle\tilde{0} \mid \phi_{k}^{F}\right\rangle & =\frac{1}{\sqrt{N}}\left[1+\frac{\Xi^{+}(z)}{\eta^{+}(z)}\right], \\
& =\frac{1}{\sqrt{N}} \frac{\left(E_{k}-E_{0}\right)}{\eta^{+}(z)},
\end{aligned}
$$

where the self-energy $\Xi^{+}(z)$ is give in Eq. (88).

Substituting Eqs.(18) into Eq.(17b), we then have

$$
\begin{aligned}
& F(\Omega)=-\operatorname{Im} \frac{1}{N} \sum_{k} \frac{1}{\Omega-E_{k}+i \epsilon} \frac{1}{\eta^{+}(z) \eta^{-}(z)} \\
& \times\left[T_{d c}^{2}+T_{0 c}^{2} \frac{\left(E_{k}-E_{0}\right)^{2}}{g^{2} B^{2}}+2 T_{d c} T_{0 c} \frac{\left(E_{k}-E_{0}\right)}{g B}\right],
\end{aligned}
$$

where $\eta^{-}(z)$ is analytically continued from the lower half plane. As done in Eq.(8), transforming the summation over the wavenumber into the integral in the limit of $N \rightarrow \infty$, the 
explicit form of $F(\Omega)$ is obtained as

$$
\begin{aligned}
& F(\Omega)=\frac{g^{2} B^{2} \sqrt{B^{2}-\Omega^{2}}}{\left(B^{2}-\Omega^{2}\right)\left(\Omega-E_{0}\right)^{2}+g^{4} B^{4}} \\
& \times\left[T_{d c}^{2}+T_{0 c}^{2} \frac{\left(\Omega-E_{0}\right)^{2}}{g^{2} B^{2}}+2 T_{d c} T_{0 c} \frac{\left(\Omega-E_{0}\right)}{g B}\right] .
\end{aligned}
$$

Now we shall decompose the absorption spectrum $F(\Omega)$ into the pole and the branch point contributions. For this purpose, we first rewrite $F(\Omega)$ in terms of the contour integral by using the relation

$$
\begin{aligned}
& \frac{1}{2 \pi} \int_{-\pi}^{\pi} d k \cdots=\int_{-B}^{B} d E_{k} \rho\left(E_{k}\right) \cdots \\
& =\frac{1}{2 \pi i} \int_{-B}^{B} d E_{k} \frac{1}{g^{2} B^{2}}\left[\eta^{+}\left(E_{k}\right)-\eta^{-}\left(E_{k}\right)\right] \cdots,
\end{aligned}
$$

where $\cdots$ represents a function of $k$. By taking the limit of $N \rightarrow \infty$ and applying these relations to Eq.(19), $F(\Omega)$ may be cast into the form of a contour integral:

$$
F(\Omega)=\operatorname{Im} \frac{1}{2 \pi i} \int_{\Gamma} \frac{1}{\Omega-E_{k}+i \epsilon} \frac{1}{\eta\left(E_{k}\right)} d E_{k},
$$

where the contour $\Gamma$ is shown in Fig 3 (a). As shown in Fig 3 the contour $\Gamma$ can be deformed to that shown in Fig 3 (b), where the cross denotes the pole location at $E_{k}=z_{d}=\tilde{E}_{0}-i \gamma$. Along $\Gamma$, the solid and dashed lines are in the first and second Riemann sheets, respectively, so that $\eta\left(E_{k}\right)$ takes the corresponding value of $\eta^{+}\left(E_{k}\right)$ and $\eta^{-}\left(E_{k}\right)$ in Eq.(22), respectively.

In order to extract the pole contribution from Eq.(22), we evaluate the residue around the pole, and obtain

$$
\begin{aligned}
& F_{0}(\Omega) \\
& =\operatorname{Im} \frac{1}{2 \pi i} \int_{\text {pole }} d E_{k} \frac{1}{\Omega-E_{k}+i \epsilon} \frac{g^{2} B^{2}}{\eta^{+}\left(E_{k}\right)} \\
& \times\left[T_{d c}^{2}+T_{0 c}^{2} \frac{\left(\Omega-E_{0}\right)^{2}}{g^{2} B^{2}}+2 T_{d c} T_{0 c} \frac{\left(\Omega-E_{0}\right)}{g B}\right] \\
& =-\operatorname{Im} \frac{N_{d}}{\Omega-z_{d}}\left[T_{d c}^{2}+T_{0 c}^{2} \frac{\left(z_{d}-E_{0}\right)^{2}}{g^{2} B^{2}}\right. \\
& \left.\quad+2 T_{d c} T_{0 c} \frac{\left(z_{d}-E_{0}\right)}{g B}\right],
\end{aligned}
$$

where $N_{d}$ is given by

$$
\begin{aligned}
N_{d}^{-1} & =\frac{d}{d z}\left[z-E_{0}-\frac{g^{2} B^{2}}{N} \sum_{k} \frac{1}{\left(z-E_{k}\right)^{+}}\right]_{z=z_{d}} \\
& =1+\frac{z_{d}\left(z_{d}-E_{0}\right)}{z_{d}^{2}-B^{2}} .
\end{aligned}
$$


Subtracting the pole contribution from Eq.(22), we can write the branch point contribution as

$$
F_{1}(\Omega)=\operatorname{Im} \frac{1}{2 \pi i} \int_{\Gamma^{\prime}} d E_{k} \frac{1}{\Omega-E_{k}+i \epsilon} \frac{1}{\eta\left(E_{k}\right)},
$$

where the contour $\Gamma^{\prime}$ is depicted in Fig 3 (c). The total absorption spectrum is then decomposed to $F(\Omega)=F_{0}(\Omega)+F_{1}(\Omega)$.

Though the exponentially decaying unstable state corresponding to the pole in the second Riemann sheet cannot be identified in the Hilbert space, it is possible to identify it outside the Hilbert space. One of the authors (T.P.) et al. have shown that the Friedrichs solution can be decomposed into the unstable state $\left|\phi_{d}\right\rangle$ (and $\left\langle\tilde{\phi}_{d}\right|$ ) and the dressed field states $\left|\phi_{k}\right\rangle$ (and $\left.\left\langle\tilde{\phi}_{k}\right|\right)$ in the generalized Hilbert space [24]:

$$
\sum_{k}\left|\phi_{k}^{F}\right\rangle\left\langle\phi_{k}^{F}|=| \phi_{d}\right\rangle\left\langle\tilde{\phi}_{d}\left|+\sum_{k}\right| \phi_{k}\right\rangle\left\langle\tilde{\phi}_{k}\right|
$$

One can prove that this decomposition has one to one correspondence with the $F_{0}$ and $F_{1}$ spectral components. It should be noted that the factor $N_{d}$ in Eqs.(24) is now recognized as a normalization constant of the unstable decaying state so that $\left\langle\tilde{\phi}_{d} \mid \phi_{d}\right\rangle=1$, and then $N_{d}=\left\langle d \mid \tilde{\phi}_{d}\right\rangle\left\langle\phi_{d} \mid d\right\rangle$.

\section{B. Fano Profile}

In order to clarify the enhancement of the branch point effect due to the singularity of the density of states in the absorption spectrum, we first show in Fig 4 the calculated results for an artificial situation with $T_{0 c}=0$. We take $B=1.0$ and $T_{d c}=1.0$ in all calculations in the present work. In Fig 4 , the results for $g=0.2$ are shown for (a) $E_{0}=-0.1$ and (b) $E_{0}=-0.98$. Solid lines are the total absorption $F(\Omega)$ calculated by Eq.(20), while the dashed and the chain lines are the pole contribution $F_{0}(\Omega)$ in Eq.(23) $\left.\mathrm{b}\right)$ and the branch point contribution $F_{1}(\Omega)$ in Eq.(25), respectively. In Fig $4(\mathrm{~b})$, the spectra are magnified around $\Omega \simeq-B$, while the overall spectrum is shown in the inset.

When $1-\left|E_{0}\right| / B \gg g(\operatorname{Fig} 4(\mathrm{a}))$, the pole contribution $F_{0}(\Omega)$ is dominant in $F(\Omega)$; the $F_{1}(\Omega)$ contribution is very small except for the tiny increase around the band edges. In this

case, $N_{d} \simeq 1$ and $\left|\tilde{E}_{0}-E_{0}\right| \ll g B$ in Eq.(23) $)$, resulting in a sharp Lorentzian spectrum of $F_{0}(\Omega)$ as shown in Fig $4(a)$. The fact that $F_{0}(\Omega)$ is dominant in the entire energy region of $-B \leq \Omega \leq B$, suggests that the time evolution of the adatom localized state almost 
completely obeys the exponential decay law, i.e. the Markovian process is predominant, as mentioned in the end of the previous section.

As $E_{0}$ gets close to the band edge (Fig $\left.4(b)\right)$, the decay rate of the unstable state is nonanalytically enhanced (as discussed in $\S 2$ ) and the energy shift of $\left|\tilde{E}_{0}-E_{0}\right|$ becomes large due to the singularity in the density of states of the miniband at the band edges, as shown in Fig. 2. Therefore the spectral width of $F_{0}(\Omega)$ gets wider and the shift of the peak position from $E_{0}$ becomes visible as shown in Fig [4(b). Furthermore, it is found from Eq. (24b) that the divergence in the density of states enhances the second term in Eq.(24b), and thus $N_{d}$ is largely reduced from 1 while $\operatorname{Im}\left[N_{d}\right]$ remains negligibly small. Consequently, the relative ratio of the branch point contribution of $F_{1}(\Omega)$ to the pole contribution $F_{0}(\Omega)$ becomes larger than in Fig 4(a). As discussed at the end of the previous section, the relative increase of the branch point contribution indicates that the time separation between the exponential and the power law decays becomes obscure in the time evolution of the surviving amplitude. Therefore, the non-Markovian process with memory effect becomes significant, which maintains the quantum coherence in the decaying process. The reduction of $N_{d}\left(=\left\langle d \mid \tilde{\phi}_{d}\right\rangle\left\langle\phi_{d} \mid d\right\rangle\right)$ also indicates that the unstable state $\left|\phi_{d}\right\rangle$ (or $\left\langle\tilde{\phi}_{d}\right|$ ) contains a larger contribution from the electronic continuum components of $|k\rangle$. This suggests that the contribution of the continuum to the dressing cloud is more significant. As shown below, the persistence of quantum coherence in the decay process, or the large dressing effect, manifests the quantum interference between the different optical transition paths once the other absorption transition $T_{0 c}$ is introduced.

Next we consider the actual case in which both optical transition paths are allowed: $T_{0 c} \neq$ 0 and $T_{d c} \neq 0$. We show in Fig $[$ (a) the calculated results of $F(\Omega)$ for the same parameters as in Fig प(a) except that $T_{0 c}=1.0$ here. The pole contribution $F_{0}(\Omega)$ and the branch point contribution $F_{1}(\Omega)$ are shown in Fig $\underline{5}$ (b) and (c), respectively. All these spectra are depicted by the black solid lines. In each panel, the spectra are further decomposed into the spectral components due to the first, second, and the third terms in [] of Eq.(20) or Eq.(23) These terms are attributed to the $d$ - $d$ diagonal component, $\tilde{0}-\tilde{0}$ diagonal component, and the interference term between these two in Eq.(17b). These are shown by the red, blue, and green lines, respectively.

Introduction of $T_{0 c}$ changes the symmetric Lorentzian spectral shape of $F(\Omega)$ shown in Fig 4(a), and yields an asymmetric spectral shape around $\Omega \simeq E_{0}$ shown by the black line 
in Fig! 5 (a). As seen from Fig 5 (a), this is caused by the interference term of Eq.(20) shown by the green line in the figure. Around $\Omega \simeq \pm B$ the $F(\Omega)$ shows a sharp rise which reflects the divergence in the density of states, though $F(\Omega)$ remains finite around $\Omega \simeq \pm B$ and $F( \pm B)=0$. It can be seen in Fig $\left[\right.$ (b) that $F_{0}(\Omega)$ is the dominant contribution to $F(\Omega)$ for $\Omega \simeq E_{0}$ and we see that the antisymmetric spectral shape of the interference component of $F_{0}(\Omega)$ (green line) is the origin of the asymmetry in $F(\Omega)$. In fact, neglecting $\operatorname{Im}\left[N_{d}\right]$ in Eqs.(23), which is appropriate except for $\left|E_{0}\right|>B$, the interference term in $F_{0}(\Omega)$ is approximately given by

$$
\begin{aligned}
& F_{0}^{\text {inter }}(\Omega)=\frac{2 T_{d c} T_{0 c}}{g B} \operatorname{Re}\left[N_{d}\right] \gamma \\
& \times\left\{\frac{\Omega-\tilde{E}_{0}}{\left(\Omega-\tilde{E}_{0}\right)^{2}+\gamma^{2}}+\frac{\tilde{E}_{0}-E_{0}}{\left(\Omega-\tilde{E}_{0}\right)^{2}+\gamma^{2}}\right\} .
\end{aligned}
$$

When the condition $1-\left|E_{0}\right| / B \gg g$ is satisfied, it holds that $\operatorname{Re}\left[N_{d}\right] \simeq 1$ and $\tilde{E}_{0}-E_{0} \ll 1$. As a result, only the first term of Eq.(27) contributes to $F_{0}^{\text {inter }}$ (Fig 5 (b), green line), leading to the antisymmetric spectral shape of the interference component of $F_{0}(\Omega)$. The asymmetric spectral shape obtained when the branch point effect is neglected is represented by the well-known Beutler-Fano profile [22].

However when $E_{0}$ lies close to the band edges, $\left|\tilde{E}_{0}-E_{0}\right|$ becomes large, the second term of Eq. (27b) can no longer be neglected, which increases the interference component of $F_{0}(\Omega)$ compared to the $d$ - $d$ diagonal component. We show in Fig 6 the calculated results for the same parameters as in Fig 4(b) where $E_{0}=-0.98 B$, except that we take $T_{d c}=1.0$. As seen in Fig 6(b) the interference component of $F_{0}(\Omega)$ (green line) is enhanced compared with that in Fig [5). As mentioned above, in this case quantum coherence plays a key role in the decay process, which is clearly reflected through the enhancement of the interference effect in the absorption spectrum.

\section{SUMMARY AND DISCUSSIONS}

In the present work, we have evaluated the charge transfer rate from an adatom impurity on a $1 \mathrm{D}$ semiconductor superlattice. The decay rate is dramatically enhanced due to two square-root forms of singularities in the density of states. In the vicinity of the singularities

at either edge of the band spectrum, the decay rate becomes a nonanalytic function of the 
coupling constant $g$ at $g=0$ as it can only be expanded in powers of $g^{4 / 3}$. The time evolution of the localized adatom state is governed by the pole and branch point contributions to the decay rate, which account for the exponential and nonexponential decay, respectively.

We have demonstrated that the absorption spectrum from the inner core level of the adatom to the conduction states is an appropriate probe to observe both contributions. While the branch point effect is usually small, it becomes important when the adatom localized state is resonant with the miniband of the 1D superlattice in the vicinity of the singularity in the density of states. In the case where the branch point effect is significant, the quantum coherence in the decay process becomes exaggerated. This explains the enhancement of the quantum interference in the absorption spectrum.

The advantage of using the semiconductor superlattice is that it is easy to vary the parameters in an electronic system. Using modern nanotechnology, we can vary the parameter values widely, in order to systematically investigate the effect on the decay process. Other spectroscopic techniques, such as the resonant optical light scattering spectrum, may also be used to investigate the time evolution of the unstable state in detail, which we are now studying.

\section{Acknowledgments}

We thank Professor V. Kocharovsky, Dr. G. Ordonez, Professor W. Schieve and Professor G. Sudarshan for insightful discussions. We acknowledge the Engineering Research Program of the Office of Basic Energy Sciences at the U.S. Department of Energy, Grant No DEFG03-94ER14465 for supporting this work. This work was supported by the Grant-in-Aid for Scientific Research from the Ministry of Education, Science, Sports, and Culture of Japan.

[1] R. Nötzel and K. H. Ploog, Adv. Mater. 5, 22 (1993).

[2] S. E. Ulloa, E. Castano, and G. Kirczenow, Phys. Rev. B 41, R12350 (1990).

[3] R. J. Haug, J. M. Hong, and K. Y. Lee, Surf. Sci. 263, 415 81992).

[4] M. S. Gudiksen et al., Nature, 415, 617 (2002).

[5] L. P. Kouwenhoven et al., Phys. Rev. Lett. 65, 361 (1990) 
[6] K. Nikolić and R. Sordan, Phys. Rev. B 58, 9631 (1998).

[7] Y. S. Joe et al., J. Appl. Phys. 88, 2704 (2000).

[8] L. van Hove, Phys. Rev. 89, 1189 (1953).

[9] A. Messica et al., Phys. Rev. Lett. 78, 705 (1997), D. Menashe and B. Laikhtman, J. Phys. Condensed Matter 10, 7793 (1998).

[10] Surface Electron Transfer Processes, ed. by R. J. D. Miller (VCH, New York, 1995).

[11] D. M. Newns, Phys. Rev. 178, 1123 (1969).

[12] T. Petrosky, Chu-Ong Ting and Sterling Garmon, Phys. Rev. Lett. 94, 043601 (2005).

[13] L. A. Khalfin, Sov. Phys. JETP 6, 1053 (1958).

[14] S. R. Wilkinson, C. F. Bharucha, M. C. Fischer, K. W. Madison, P. R. Morrow, Q. Niu, B. Sundaram, and M. G. Raizen, Nature387, 575 (1997).

[15] G. Ordonez, T. Petrosky, and I. Prigogine, Phys. Rev. A63, 052106 (2001).

[16] T. Petrosky, G. Ordonez, and I. Prigogine, Phys. Rev. A64, 062101 (2001).

[17] B. Misra and E. C. G. Sudarshan, J. Math. Ohys. 18, 756 (1977).

[18] W. M. Itano, D. J. Heinzen, J. J. Bollinger, and D. J. Wineland, Phys. Rev. A41, 2295 (1990).

[19] T. Petrosky, S. Tasaki, I. Prigogine, Physica A170, 306 (1991).

[20] K. Koshino and A. Shimizu, Phys. Rep. 412, 191 (2005).

[21] K. Friedrichs, Commun. Pure Appl. Math. 1, 361 (1948).

[22] U. Fano, Phys. Rev. 15, 1866 (1961).

[23] Y. Zheng and T. Ando, Phys. Rev. B66, 085328 (2002).

[24] T. Petrosky, I. Prigogine, and S. Tasaki, PhysicaA 173, 175 (1991). 
FIG. 1: (a) An adatom attached to a 1D quantum-dot array, and (b) level structures of the adatom localized state and the bound state in each quantum well. The width of each well is a few nm to $100 \mathrm{~nm}$. The adatom is located at the $n=0$-th well. The two optical absorption transitions from the core level $|c\rangle$ with $T_{d c}$ and $T_{0 c}$ are also shown by the arrows.

FIG. 2: (a) $E_{i} / B$ and $\tilde{E}_{0} / B$ vs. $E_{0} / B$ for $g=0.5$, which are shown by the thick solid and dashed lines, respectively. The location of the critical values $\pm E_{\gamma} / B$ are indicated by the arrows. The thin line is $y=E_{0} / B$. (b) $\gamma / B$ vs. $E_{0} / B$ for $g=0.1$. The maximum value of $\gamma_{\max } / B$ occurs at $E_{0} / B= \pm 1$.

FIG. 3: The contours of the integral for $F(\Omega)$ Eq.(22) (a) and its deformation (b), and the integral for $F_{0}(\Omega)$ Eq. $(23)(\mathrm{c})$.

FIG. 4: The calculated $F(\Omega)$ (solid line), $F_{0}(\Omega)$ (dashed line), and $F_{1}(\Omega)$ (chain line) for $g=0.2$, and $T_{d c}=1.0$ and $T_{0 c}=0:$ (a) $E_{0}=-0.1 B$ and (b) $E_{0}=-0.98 B$. The thin vertical lines indicate the position of $E_{0}$. In (b), the horizontal axis is expanded around $\Omega \simeq-B$, while the overall spectrum is shown in the inset.

FIG. 5: The calculated $F(\Omega)(\mathrm{a}), F_{0}(\Omega)(\mathrm{b})$, and $F_{1}(c)$ for the same parameters for Fig 5 (a) except $T_{0 c}=1.0:$ The spectra are decomposed into the $d$ - $d$ diagonal (red line), 0 -0 diagonal (blue line), and the interference terms (green line).

FIG. 6: The parameters here are the same as in Fig [5) except that $T_{0 c}=1.0$. 


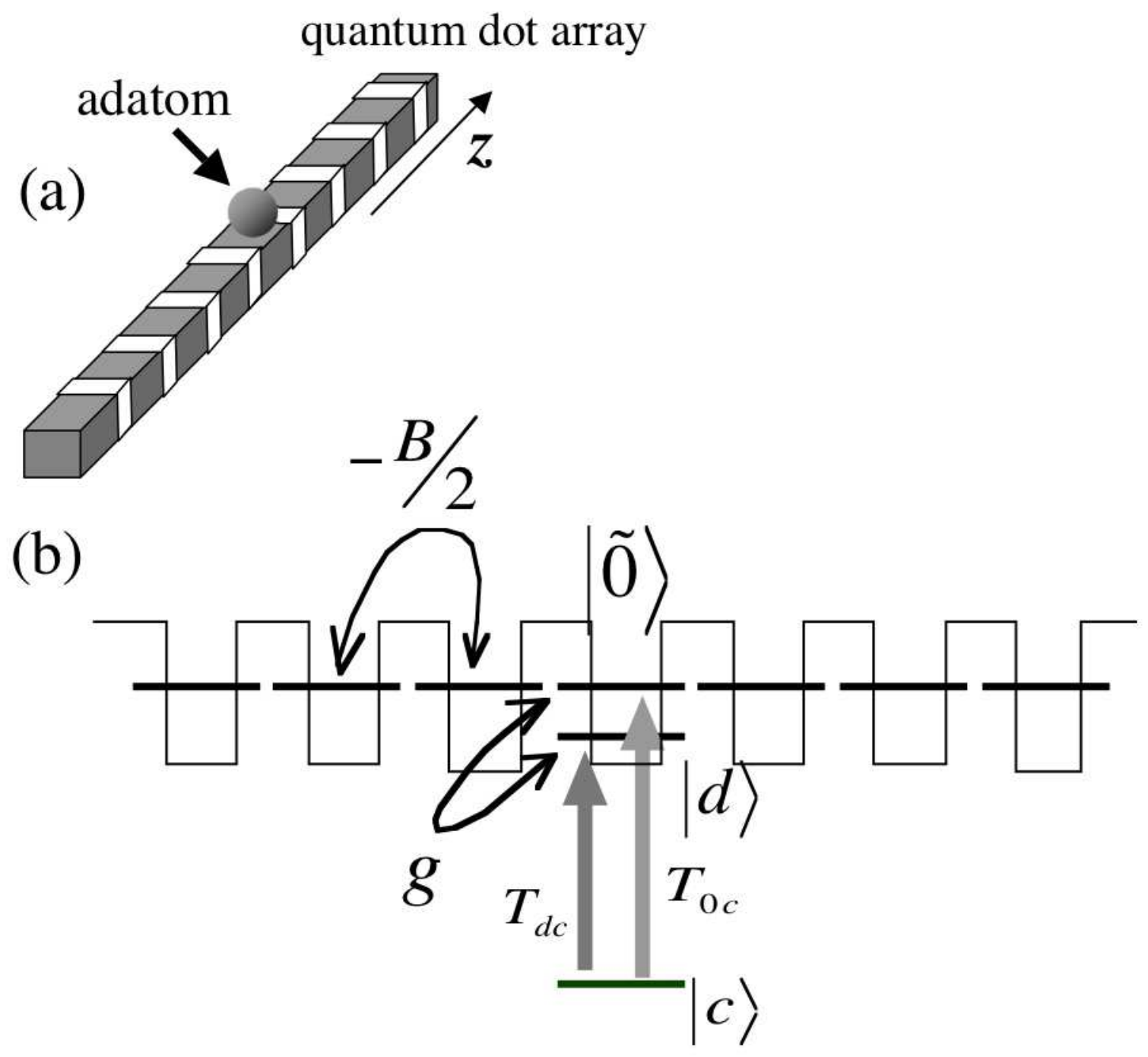



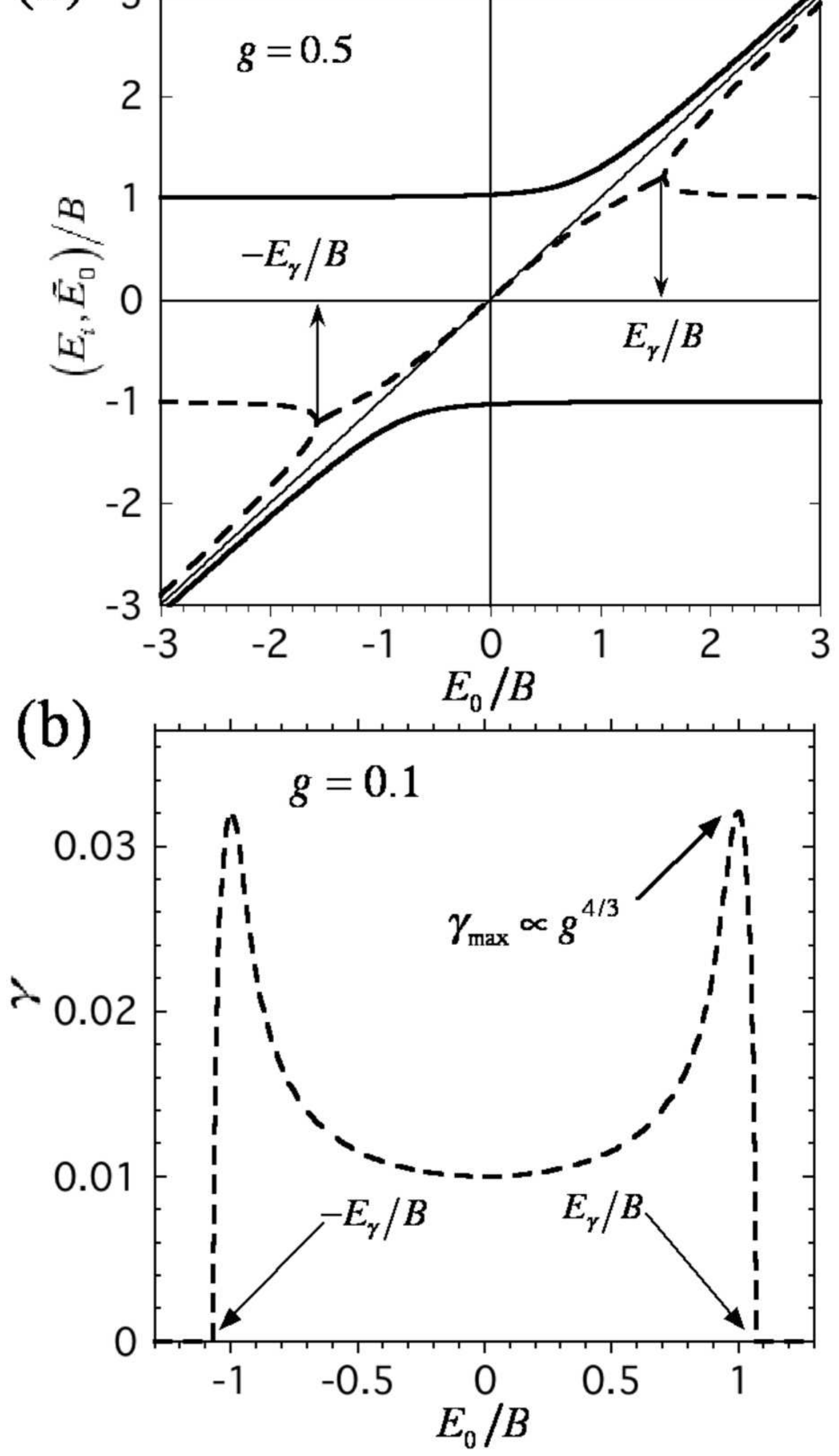


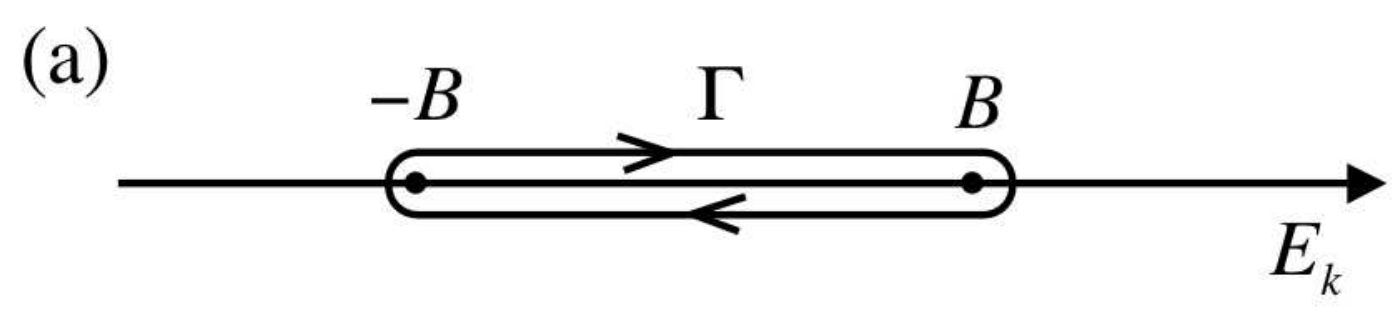

(b)

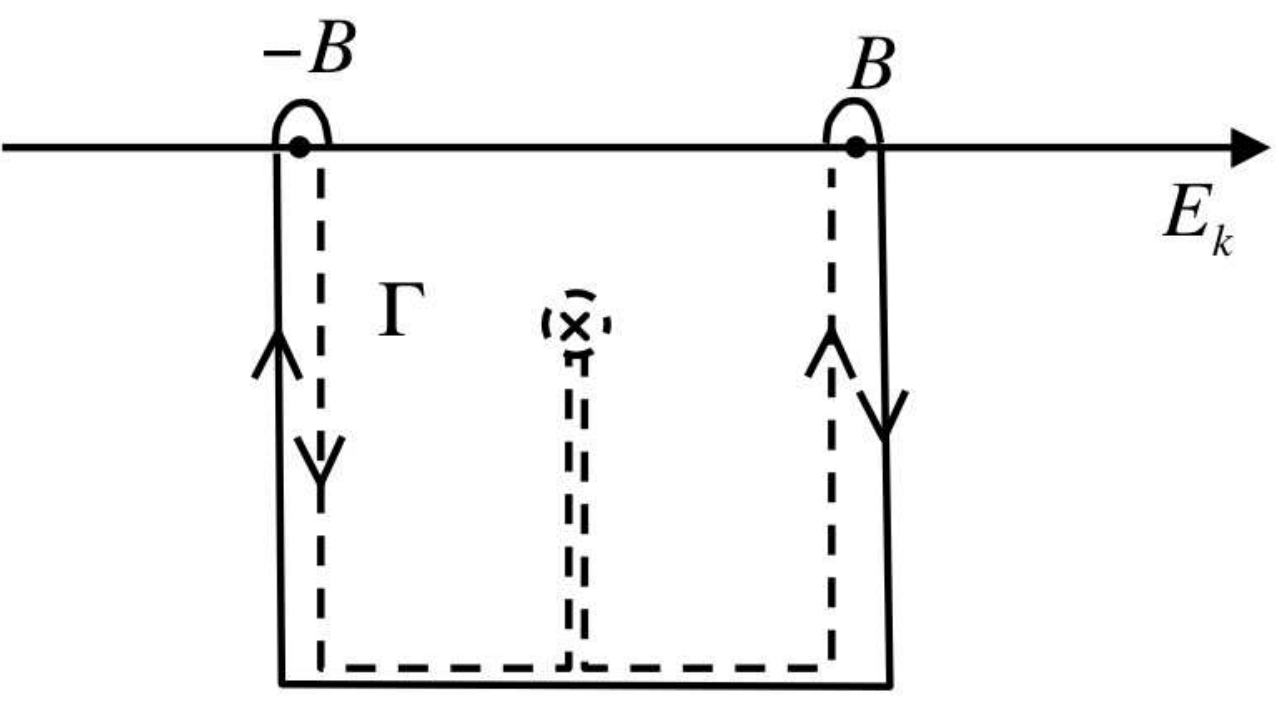

(c)

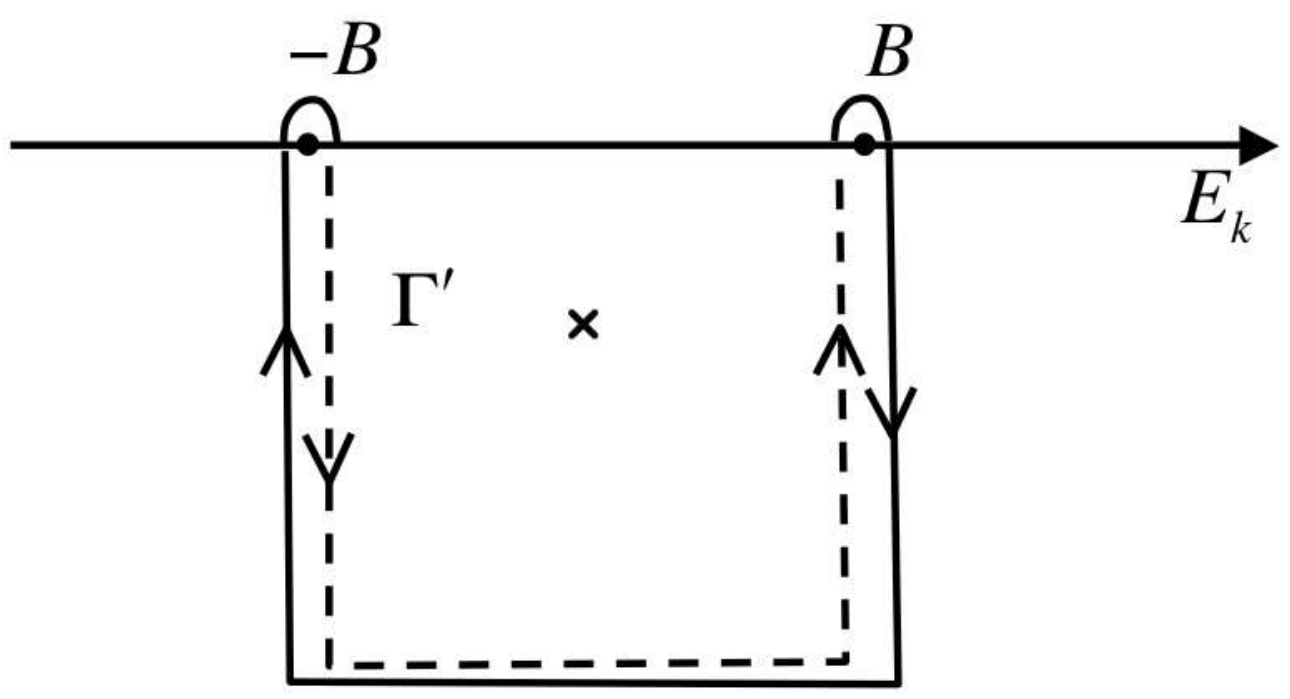



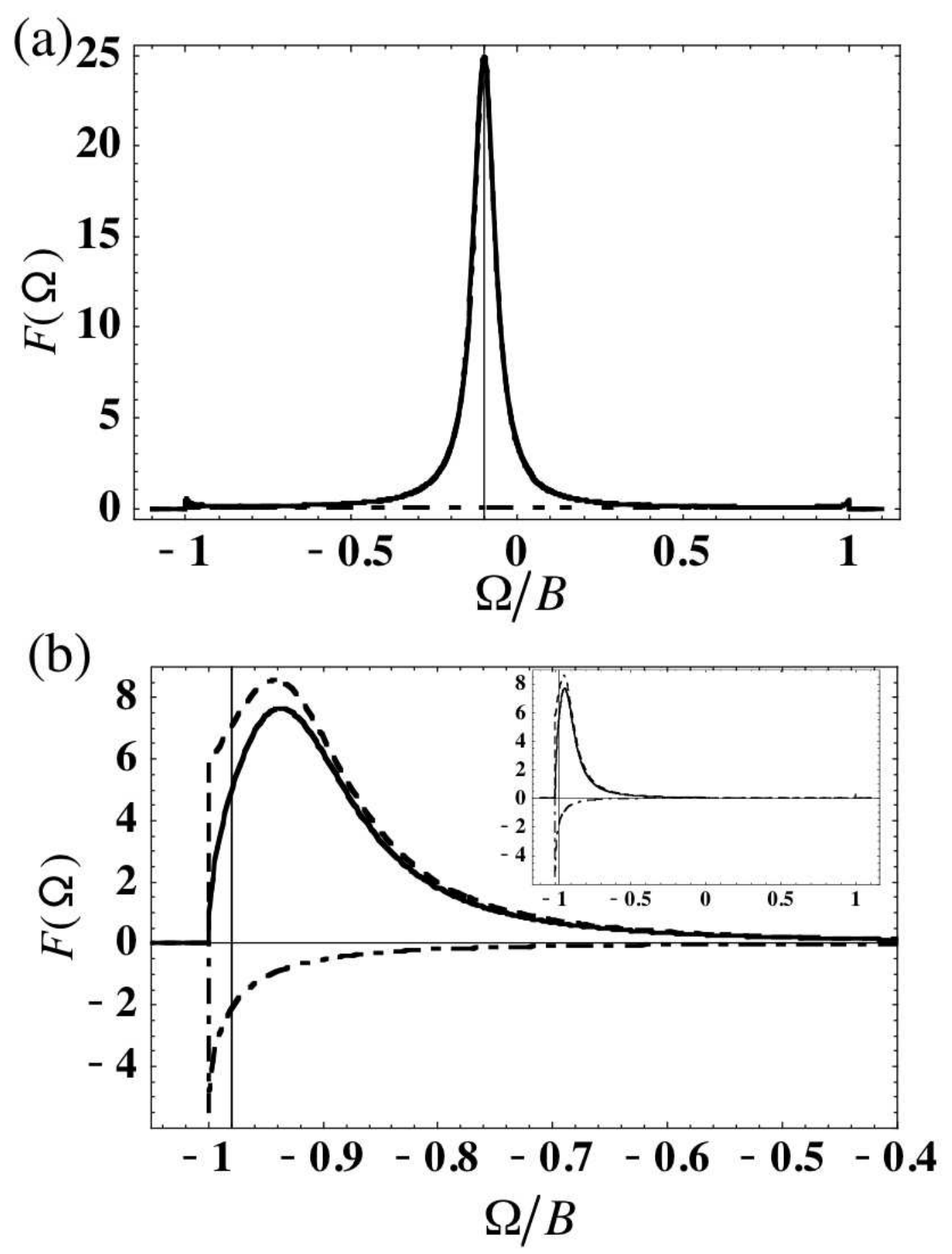

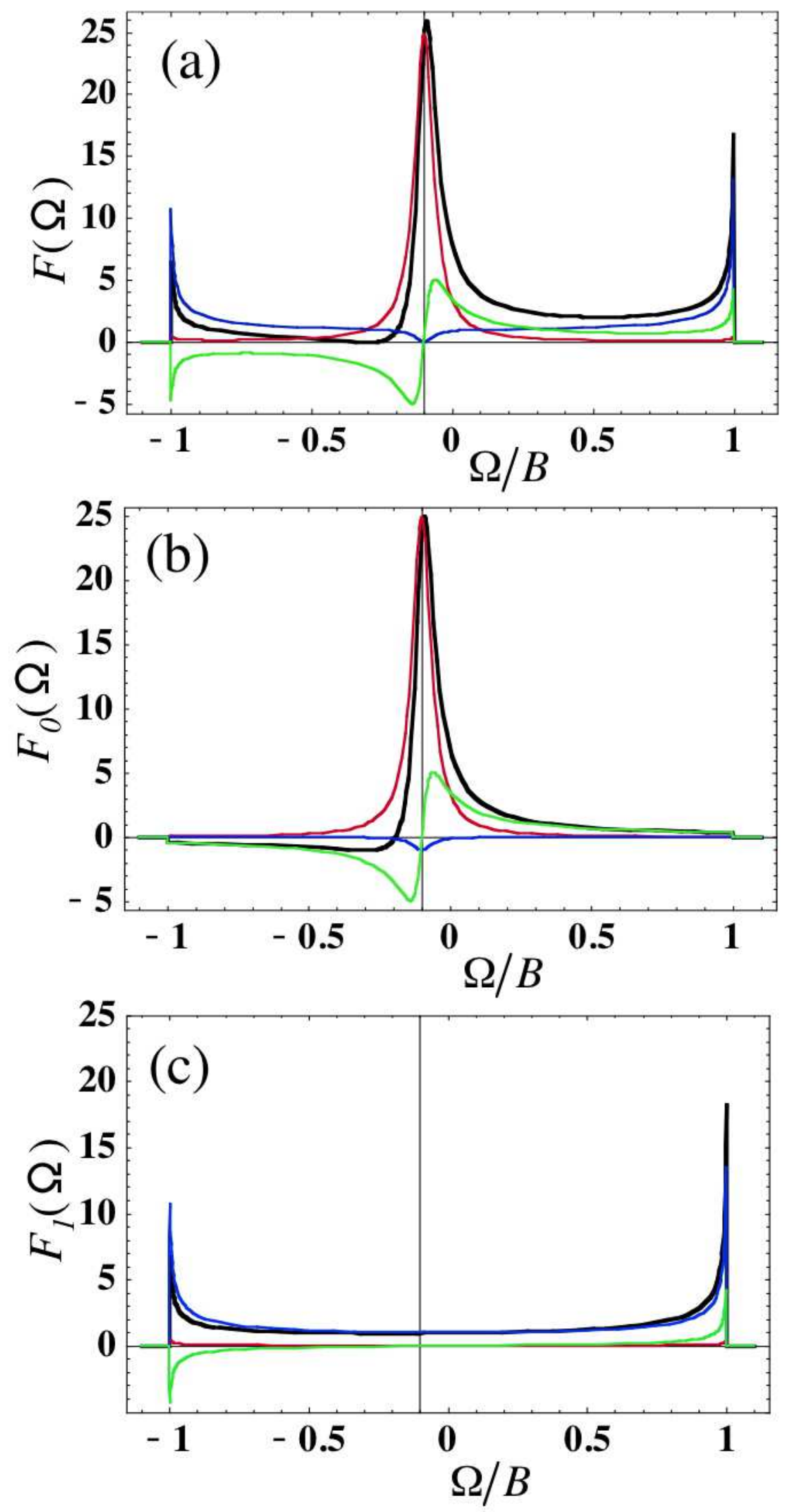

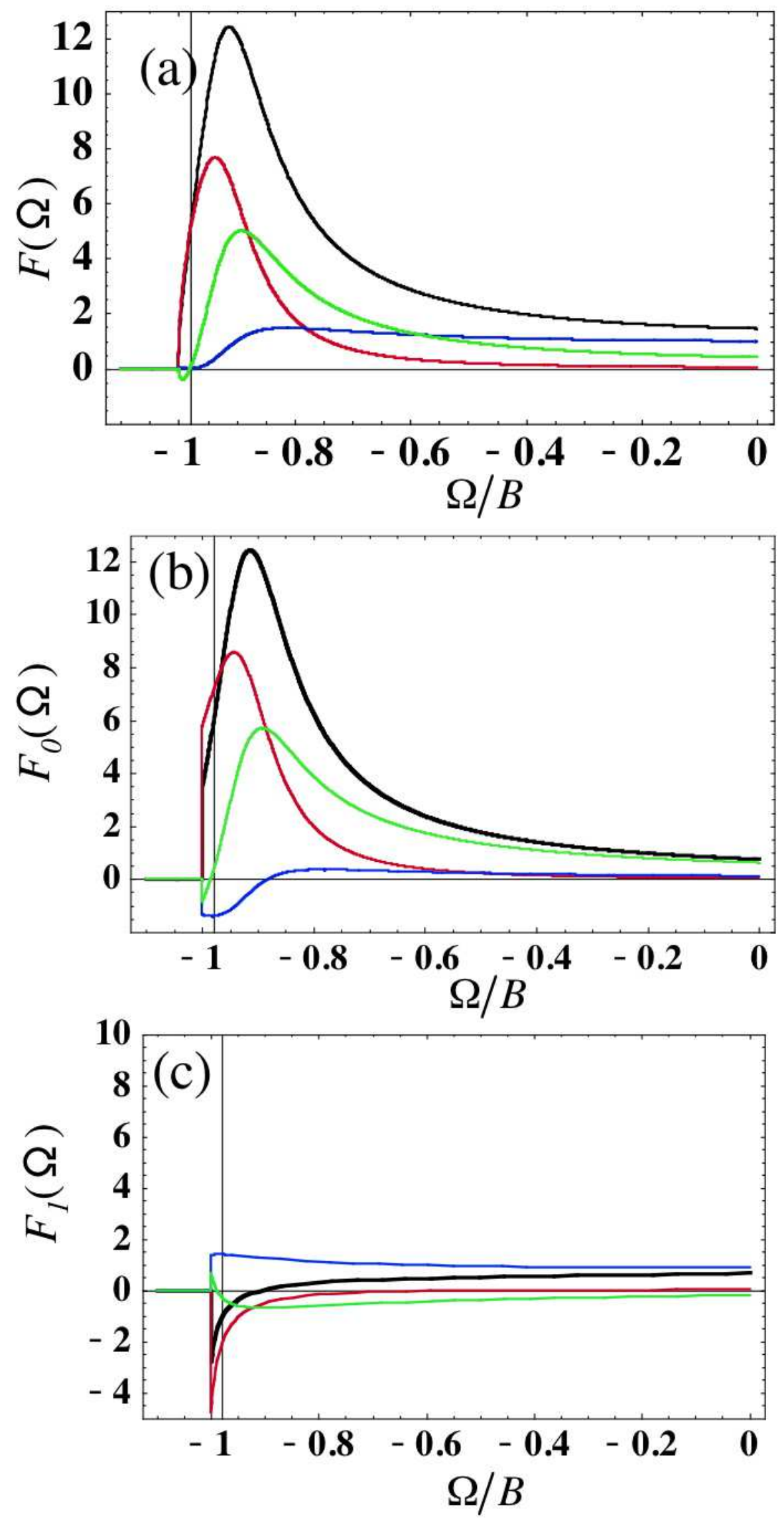